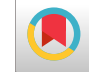

\title{
Reassessing the Risks of MMR Vaccination for Egg-Allergic Patients Referred by Healthcare Workers; a Clinical Paradigm
} \author{
Ceren Can $^{1, " *}$ and Bahar Kural ${ }^{2}$ \\ ${ }^{1}$ Department of Pediatric Immunology and Allergy, Bakırköy Dr. Sadi Konuk Training and Research Hospital, Istanbul, Turkey \\ ${ }^{2}$ Department of Pediatrics, Bakırköy Dr. Sadi Konuk Training and Research Hospital, Istanbul, Turkey \\ "Corresponding author: MD, Department of Pediatric Immunology and Allergy, Bakırköy Dr. Sadi Konuk Training and Research Hospital, Istanbul, Turkey. Tel: +90-2124147171, \\ Fax:+90-2124146494, Email: cereni35@yahoo.com
}

Received 2019 February 05; Revised 2019 May 20; Accepted 2019 May 26.

\begin{abstract}
Background: Egg allergy for the Measles-Mumps-Rubella (MMR) vaccine is still the cause of concern for many healthcare workers and families due to reactions such as anaphylaxis.

Objectives: This study aimed to reassess the risk of MMR vaccination in infants with egg allergy referred by healthcare workers.

Methods: This cross-sectional observational study was performed in a university-affiliated hospital in Istanbul, Turkey, between March 2017 and September 2018. The study patients were one-year-old infants who were candidates for MMR vaccination, and referred to our outpatient allergy clinic by healthcare workers due to egg allergy. The children were diagnosed with egg allergy by a pediatric allergist and then received the MMR vaccine.

Results: Among 50 children aged one year, 19 (38\%) were female, and 31 (62\%) were male. The diagnosis was atopic dermatitis in 29 (58\%) patients, urticaria in 18 (36\%) patients, angioedema in $2(4 \%)$ patients, and anaphylaxis in one (2\%) patient. Fourteen (28\%) patients had both egg and cow's milk allergy. Four ( $8 \%$ ) patients had egg, cow's milk, and wheat allergy. The total IgE level was 119.80 $\pm 213.43 \mathrm{IU} / \mathrm{mL}$. Specific IgE levels for egg white were positive in 37 patients. Specific IgE levels for egg white were $15.26 \pm 29.64 \mathrm{kU} / \mathrm{L}$. Skin prick test results were positive for egg allergens in 35 patients with a mean diameter of $3.12 \pm 2.18 \mathrm{~mm}$ for egg yolk and $3.27 \pm$ $2.62 \mathrm{~mm}$ for egg white. None of the patients with egg allergy developed anaphylactic reaction and only one patient had urticaria within 5 min after vaccination. There was no correlation between specific IgE levels for egg white and diagnosis of the patients.

Conclusions: MMR vaccine administration to children with egg allergy is safe. Life-threatening reactions to the MMR vaccine in patients with egg-allergy are very rare. It seems in cases of hypersensitivity reactions such as acute anaphylaxis events, the MMR vaccine needs to be administered in a hospital under the supervision of a pediatric allergist.
\end{abstract}

Keywords: Anaphylaxis, Angioedema, Atopic Dermatitis, Children, Cow's Milk Allergy, Egg Allergy, Immunoglobulin E, Measles-Mumps-Rubella Vaccine, Urticaria, Vaccination.

\section{Background}

Vaccination side effects and fear of anaphylaxis in specific conditions such as egg allergy may cause a delay in vaccination based on the decision of healthcare workers and families. Therefore, it has become necessary to confirm the vaccination status of children with egg allergy. Hen's egg allergy is the second most common food allergy in childhood (1). Of young children, $1 \%-2 \%$ are affected by egg allergies $(2,3)$. Five major allergenic proteins are responsible for immunoglobulin $\mathrm{E}$ (IgE)-mediated reactions. Although ovomucoid is the dominant allergen in eggs, ovalbumin is the most abundant protein in hen's egg white $(4,5)$. The management of egg allergy involves the avoidance of egg-containing products, education in the appropriate management of accidental exposures, and following up for resolution of the allergy (6). Most cases are resolved during childhood or adolescence (7).
The Measles-Mumps-Rubella (MMR) vaccine is a live, attenuated vaccine that is derived from Enders' attenuated Edmonston strain propagated in chick embryo cell culture, Jeryl Lynn ${ }^{\mathrm{TM}}$ (B level) strain of mumps virus propagated in chick embryo cell culture, and Wistar RA 27/3 strain of live attenuated rubella virus propagated in WI38 human diploid lung fibroblasts. It is licensed for routine use in individuals aged $\geq 12$ months (8). The MMR vaccine is included in the pediatric immunization schedule in Turkey and administered at 12 months through four to six years of age (9). The measles vaccine is produced in chicken embryo cell culture and does not include significant amounts of egg white protein (10). Egg allergy does not contraindicate the MMR vaccine. Hypersensitivity reactions to MMR are usually minor (urticaria) and are attributed to the trace amounts of neomycin or gelatin but not to egg antigens (11-14). The controversy remains among 
healthcare workers concerning MMR and egg allergy due to anaphylactic reactions although the concentrations of processed chicken egg protein in the vaccine are too low to trigger an allergic reaction (15). Despite various recommendations and guidelines for children with egg allergies, the children are still directed to administer the MMR vaccine in the hospital environment (16). It is reported that not only healthcare workers but also the parents are extremely anxious and tend to make vaccination at the hospital (17) In the United Kingdom, it has been reported that in the past years, children with egg allergy had the possibility of being neglected or delayed for the MMR vaccine but this tendency has improved with the clarity of guidelines and dissemination of correct information (18).

\section{Objectives}

In this study, we aimed to reassess the risks of MMR vaccination and allergic reactions in infants with egg allergy.

\section{Methods}

This cross-sectional observational study was performed in the outpatient clinic of a pediatric allergy department at a university-affiliated hospital in Istanbul, Turkey, between March 2017 and September 2018. Our center is a governmental and reference hospital. All of the babies included in the study were referred to the allergy outpatient clinic by a healthcare worker because of the suspicion of egg allergy before vaccination. Infants who were not referred by healthcare workers due to egg allergy were excluded from the study. All patients were diagnosed with immunoglobulin E (IgE)-mediated egg allergy in the allergy outpatient clinic.

\subsection{Data Collection}

The diagnosis of egg allergy in patients with the symptoms after egg intake (IgE-mediated) was done with the removal of food from the diet, and improvement of symptoms, egg-white-specific IgE measurement and skin prick test. All of the babies were 12-months-old and were not previously vaccinated with MMR. The vaccine strains were $M-$ M-RII (Merck, West Point, PA). The gender, diagnosis, presence of other food allergies, total IgE, specific IgE levels of egg white (F1), skin prick test results, and allergic reactions after vaccination were retrospectively recorded from the patients' files. The MMR vaccines were administered subcutaneously with 23 to 25-gauge needles to the lateral thigh in the hospital emergency room by the same researcher. Patients were observed at least one hour after vaccination (Figure 1).
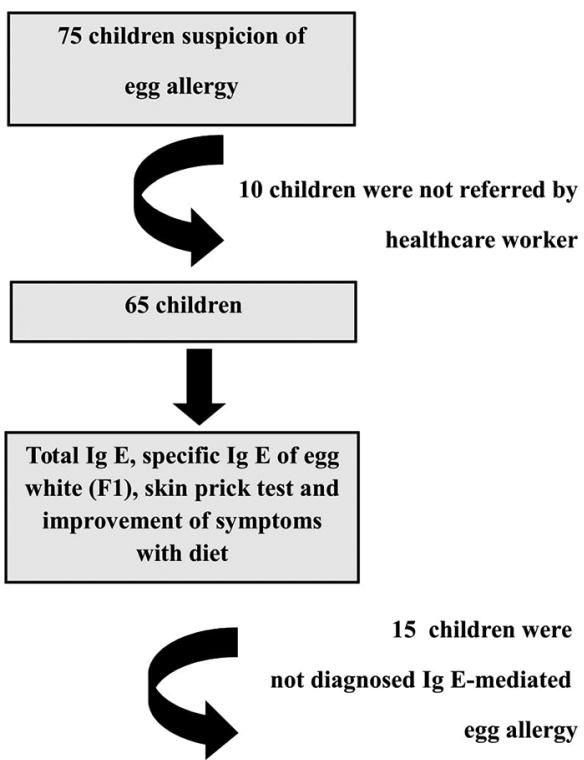

15 children were not diagnosed Ig E-mediated egg allergy

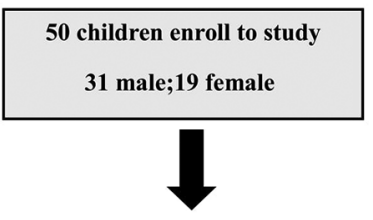

MMR vaccine administered (S.C) in hospital and observed $>1$ hours

Figure 1. Study process of participants' enrollment

\subsection{Measurements}

The serum total IgE levels were measured by Chemiluminescent Immunometric Assay (CLIA), (Immulite 2000 Allergy; Diagnostic Products Corp., Los Angeles, USA). Specific IgE antibodies (egg white) were detected qualitatively by Chemiluminescent Enzyme-Labeled Immunoassay (Diagnostic Products Corporation, Los Angeles, USA). The cutoff value for the egg white serum specific IgE level was $0.35 \mathrm{kU} / \mathrm{L}$. A skin prick test was performed for food allergens (milk, egg white, egg yolk, wheat, peanut, cacao, tuna, strawberry, and tomato [Allergopharma Joachim Ganzer $\mathrm{KG}$, Germany]). A positive skin prick test was accepted by the presence of wheal width of $\geq 3 \mathrm{~mm}$ for egg white and/or egg yolk by the same researcher. The study protocol was approved by the local Ethics Committee of the hospital, and written informed consent from parents was obtained for each child prior to the study. All procedures performed in studies involving human participants were in accordance with the ethical standards of the institutional and/or national research committees and based on 
the 1964 Helsinki Declaration and its later amendments or comparable ethical standards. Ethical approval number/date was 2018-14-19/06.08.2018.

\subsection{Statistical Analysis}

Statistical analyses were performed using the Number Cruncher Statistical System (NCSS) 2007 statistical software (Utah, USA). Descriptive indices were calculated using descriptive statistical methods (mean and standard deviation), and numbers and percentages were used for expressing frequencies of patients. Correlation between specific $\operatorname{IgE}$ levels for egg white and diagnosis of the patients was assessed using the Pearson correlation test. Statistical significance was defined at a P value of less than 0.05 .

\section{Results}

In this study, a total of 75 children with a suspicion of allergy to eggs were evaluated. As 10 children were not referred by healthcare workers and 15 children were not diagnosed with IgE-mediated egg allergy, they were not included in the study (Figure 1). The general characteristics of allergic cases are shown in Table 1 . All the children were aged one year. Of the patients, 19 (38\%) were female and 31 (62\%) were male. Patient diagnosis, as shown in Table 2, was atopic dermatitis in 29 (58\%) patients, urticaria in 18 (36\%) patients, angioedema in two (4\%) patients, and anaphylaxis in one (2\%) patient.

\begin{tabular}{|c|c|c|}
\hline & Female $(N=19)$ & $\operatorname{Male}(\mathbf{N}=\mathbf{3 1})$ \\
\hline \multicolumn{3}{|l|}{ Causes of allergy } \\
\hline Egg & $14(73.6)$ & $18(51)$ \\
\hline Egg + cow's milk & $5(26.4)$ & $9(29)$ \\
\hline Egg + cow's milk + wheat & 0 & $4(20)$ \\
\hline \multicolumn{3}{|l|}{ Skin prick test results } \\
\hline Egg white & $11(57.8)$ & $13(41.9)$ \\
\hline Negative & $5(26.4)$ & $10(22.5)$ \\
\hline Whole egg & $3(15.8)$ & $7(32.4)$ \\
\hline Egg yolk & 0 & $1(3.2)$ \\
\hline
\end{tabular}

${ }^{\mathrm{a}}$ Values are expressed as No. (\%).

Fourteen (28\%) patients had both egg and cow's milk allergy. Four (8\%) patients had egg, cow's milk, and wheat allergy. The total mean IgE level was $119.80 \pm 213.43 \mathrm{IU} / \mathrm{mL}$. The specific IgE levels for egg white were $15.26 \pm 29.64 \mathrm{kU} / \mathrm{L}$. Skin prick test results were positive for egg allergens in 35 patients with a mean diameter of $3.12 \pm 2.18 \mathrm{~mm}$ for egg yolk and $3.27 \pm 2.62 \mathrm{~mm}$ for egg white.

\begin{tabular}{lcc}
\hline Table 2. Diagnosis of the Patients $^{\mathrm{a}}$ & & \\
\hline Diagnosis & Female $(\mathbf{N}=\mathbf{1 9})$ & Male $(\mathbf{N}=\mathbf{3 1})$ \\
\hline Atopic dermatitis & $12(63.1)$ & $17(54.8)$ \\
Urticaria & $8(42.1)$ & $10(32)$ \\
Anaphylaxis & 0 & $1(3.2)$ \\
Angioedema & 1 & $1(3.2)$ \\
\hline
\end{tabular}

${ }^{\mathrm{a}}$ Values are expressed as No. (\%).

Among all infants only one patient had urticaria within 5 min after vaccination. It resolved with pheniramine maleate treatment in one hour. She has had atopic dermatitis since one-month-old. At seven-months-old, she experienced urticaria after egg consumption. Her specific IgE level for egg white was 3.2 KU/L. Her skin prick test showed a diameter of $5.2 \mathrm{~mm}$ for the egg white. None of the patients with egg allergy developed anaphylactic reactions. There was no correlation between specific IgE levels for egg white and diagnosis of the patients (Table 3 ).

\begin{tabular}{|c|c|c|}
\hline \multirow[t]{2}{*}{ Diagnosis } & \multicolumn{2}{|c|}{ Specific IgE Positive Patients for Egg White $(\mathrm{N}=37)$} \\
\hline & Pearson Correlation & P Value \\
\hline Atopic dermatitis & 0.147 & 0.08 \\
\hline Urticaria & 0.125 & 0.11 \\
\hline Anaphylaxis & 0.07 & 0.24 \\
\hline Angioedema & 0.07 & 0.24 \\
\hline
\end{tabular}

\section{Discussion}

Since the onset of MMR vaccine administration, the vaccination of individuals with egg allergies has been the subject of controversy (19). Even though many reports have shown that egg-sensitive individuals can safely take the MMR vaccine, their vaccination is still postponed or rejected (20). The vaccine strains of M-M-RII (Merck, West Point, PA), accounting for one of the commonly used MMR vaccines, are grown in chick embryo cells and human diploid cell culture. Since the vaccine is not manufactured in eggs, no egg protein is found in the vaccine or the amount is usually insufficient to cause allergic reactions. However, due to the persistence of doubt, it is a common practice to advise parents to expose their children to the egg before MMR immunization. Then, children are referred to allergists if any form of hypersensitivity reaction is observed (15)

Parents of children with allergies to egg proteins and healthcare workers who follow up them are careful and 
concerned about allergic reactions after MMR vaccination. In observational studies, it is stated that concerns are out of place as there are no allergic reactions to egg proteins found in the MMR vaccine (20). The reliability of MMR vaccination in egg-allergic individuals has been assessed in several studies. Andersen et al. reported the administration of 41 doses of the MMR vaccine to 32 egg-sensitive patients. None showed an anaphylactic/allergic reaction to the MMR vaccine (21). In the UK, 200 egg-allergic children were skin prick tested with the MMR or measles vaccine. Five of the patients had positive reactions, one of whom exhibited anaphylaxis after the intradermal test. The remaining 199 patients were vaccinated without side effects (22). Eleven of 14 patients with suspected egg allergies at 18 - 43 years of age were evaluated with the radioallergosorbent test (RAST) and egg white-specific IgE. Three of them were given intradermal test doses. Of these, urticaria was immediately developed only in one RAST positive person. Another RAST positive person also developed a small local reaction. Duncan et al. concluded that people with egg allergies can be vaccinated without excessive risk under properly controlled and monitored conditions (23). GoodyearSmith et al. reported that 73 children received MMR without any side effects in the primary care (24).

In addition, the MMR vaccine was shown to cause one case of anaphylaxis, one urticaria, and 0.3 asthma symptoms in 100000 injections (25). The only reaction observed in our study was urticaria. No severe adverse reaction was seen; none of the patients had anaphylaxis.

Although the MMR vaccine is reported to be safe for patients with egg allergy, there are cases with anaphylaxis developing after MMR vaccine administration (26). Most severe allergic reactions to MMR are seen in children who do not have egg allergies. Sensitivity to other vaccine components, like gelatin or neomycin, accounts for many reactions. If a child is not vaccinated against measles, $\mathrm{s} / \mathrm{he}$ can come across its potentially lethal sequelae (27). Current thinking favors vaccination in spite of egg allergy; therefore, vaccination should be performed at a center where patients are observed after vaccination and anaphylaxis treatment is carried out if necessary $(26,28)$. No or delayed vaccination cannot be justified because it puts the child at risk of a serious infection. A careful approach is recommended in cases of anaphylactic reaction after egg consumption or previous vaccinations. Expert evaluation of these cases and vaccination in a supervised environment are required (27).

The hospital application of MMR vaccination imposes a burden on hospital expenditures and leads to unwanted concerns in parents. Due to concerns about egg allergy, a hospital-based study in Ireland reported that $32 \%$ of children were referred to hospital clinics for routine immu- nization within a year for the MMR vaccine (29). In our study, the MMR vaccine was given to infants referred to the hospital with expert guidance. Despite the current positive safety studies, it is difficult to achieve a change in daily practice.

There were some limitations in our study. First, the number of cases was limited because the study included only one-year-old children who were referred by healthcare workers (note that the first time of MMR vaccination is at one year of age according to the Turkish Ministry of Health vaccination schedule). The utilized sample size $(\mathrm{n}=$ 50) was not quite large and it should be considered in the interpretation of the results. Second, there was no specification study to determine which egg proteins (ovalbumin and/or ovomucoid) caused sensitization in the studied children.

In summary, our study supports that MMR vaccine administration to children with egg allergy is safe. Lifethreatening reactions to MMR vaccination in patients with egg-allergy are very rare. We believe that in cases of hypersensitivity reactions such as anaphylaxis the MMR vaccine needs to be administered in a hospital under the supervision of a pediatric allergist.

\section{Footnotes}

Authors' Contribution: Substantial contributions to conception and design, acquisition of data or analysis and interpretation of data, drafting the article and final approval of the version to be published: Ceren Can and Bahar Kural.

Conflict of Interests: The authors declare no conflict of interests.

Ethical Approval: Ethical approval number/date: 2018-1419 / 06.08.2018.

Funding/Support: This study was not funded.

\section{References}

1. Rona RJ, Keil T, Summers C, Gislason D, Zuidmeer L, Sodergren E, et al. The prevalence of food allergy: A meta-analysis. J Allergy Clin Immunol. 2007;120(3):638-46. doi: 10.1016/j.jaci.2007.05.026. [PubMed: 17628647].

2. Sicherer SH, Sampson HA. 9. Food allergy. J Allergy Clin Immunol. 2006;117(2 Suppl Mini-Primer):S470-5. doi: 10.1016/j.jaci.2005.05.048. [PubMed: 16455349].

3. Eggesbo M, Botten G, Halvorsen R, Magnus P. The prevalence of allergy to egg: A population-based study in young children. Allergy. 2001;56(5):403-11. [PubMed: 11350303].

4. Heine RG, Laske N, Hill DJ. The diagnosis and management of egg allergy. Curr Allergy Asthma Rep. 2006;6(2):145-52. [PubMed: 16566865].

5. Suzuki M, Fujii H, Fujigaki H, Shinoda S, Takahashi K, Saito K, et al. Lipocalin-type prostaglandin D synthase and egg white cystatin react with IgE antibodies from children with egg allergy. Allergol 
Int. 2010;59(2):175-83. doi: 10.2332/allergolint.09-OA-0121. [PubMed: 20179418].

6. Clark AT, Skypala I, Leech SC, Ewan PW, Dugue P, Brathwaite N, et al. British Society for Allergy and Clinical Immunology guidelines for the management of egg allergy. Clin Exp Allergy. 2010;40(8):1116-29. doi: 10.1111/j.1365-2222.2010.03557.x. [PubMed: 20649608].

7. Savage JH, Matsui EC, Skripak JM, Wood RA. The natural history of egg allergy. J Allergy Clin Immunol. 2007;120(6):1413-7. doi: 10.1016/j.jaci.2007.09.040. [PubMed: 18073126].

8. Merck. 2019, [cited 2019 Feb]. Available from: https://www.merck. com/product/usa/pi_circulars/m/mmr_ii/mmr_ii_pi.pdf.

9. Turkish Ministry of Health. General Directorate of Primary Health Care Services, Extended Immunization Program Circular. Ankara; 2018, [cited 2018 Dec 15]. Available from: https://www.saglik.gov.tr/TR, 11080/ genisletilmis-bagisiklama-programi-genelge.

10. American Academy of Pediatrics. Measles.in : Kimberlin DW, Brady MY, Hackson MA, editors. Red Books: 2015 Report of the Committee on Infectious Diseases. 30th ed. Elk Grove Village, IL: American Academy of Pediatrics; 2015.

11. Fasano MB, Wood RA, Cooke SK, Sampson HA. Egg hypersensitivity and adverse reactions to measles, mumps, and rubella vaccine. $J P e-$ diatr. 1992;120(6):878-81. [PubMed: 1593346].

12. Freigang B, Jadavji TP, Freigang DW. Lack of adverse reactions to measles, mumps, and rubella vaccine in egg-allergic children. Ann Allergy. 1994;73(6):486-8. [PubMed: 7998661].

13. Aickin R, Hill D, Kemp A. Measles immunisation in children with allergy to egg. BMJ. 1994;309(6949):223-5. doi: 10.1136/bmj.309.6949.223. [PubMed: 8069138]. [PubMed Central: PMC2540766].

14. Pool V, Braun MM, Kelso JM, Mootrey G, Chen RT, Yunginger JW, et al. Prevalence of anti-gelatin IgE antibodies in people with anaphylaxis after measles-mumps rubella vaccine in the United States. Pediatrics. 2002;110(6). e71. doi: 10.1542/peds.110.6.e71. [PubMed: 12456938].

15. See TM, Jin TE, Peng HC, Chiee AYA. Measles-Mumps-Rubella vaccine for children with egg allergy: Is admission for inpatient vaccination necessary. Med J Malaysia. 2016;71:157-60.

16. Bandi S, Macdougall C. MMR and egg allergy: To vaccinate or not to vaccinate? Br J Gen Pract. 2010;60(578):693-4. doi: 10.3399/bjgp10X515511. [PubMed: 20849703]. [PubMed Central: PMC2930231].

17. Govindaraj P, Alfaham M, Davies C, Tuthill D. Decline of hospital admissions for MMR vaccinations in children with egg allergy. Arch
Dis Child. 2009;94(11):914-5. doi: 10.1136/adc.2009.166371. [PubMed: 19847006].

18. Fox AT, Swan KE, Perkin M, du Toit G, Lack G. The changing pattern of measles, mumps and rubella vaccine uptake in egg-allergic children. Clin Exp Allergy. 2014;44(7):999-1002. doi: 10.1111/cea.12325. [PubMed: 24750550].

19. Khakoo GA. Recommendations for using MMR vaccine in children allergic to eggs. Bmj. 2000;320(7239):929-32. doi: 10.1136/bmj.320.7239.929.

20. Czajka H, Czajka S, Dylag KA, Borek E, Kuchar E. Vaccination against measles, mumps, and rubella in the light of current epidemic threats: Unjustified postponement. Adv Exp Med Biol. 2019;1153:101-7. doi: 10.1007/5584_2019_344.[PubMed:30758772].

21. Andersen DV, Jorgensen IM. MMR vaccination of children with egg allergy is safe. Dan Med J. 2013;60(2). A4573.

22. Baxter DN. Measles immunization in children with a history of egg allergy. Vaccine. 1996;14(2):131-4. doi: 10.1016/0264-410x(95)00154-s.

23. Duncan CJ, McCallum AD, Macdonald R, Jones ME. Vaccination of people with suspected egg allergy is safe and feasible. BMJ. 2011;343:d5780. author reply d5783. doi: 10.1136/bmj.d5780. [PubMed: 21914744].

24. Goodyear-Smith F, Wong F, Petousis-Harris H, Wilson E, Turner N. Follow-up of MMR vaccination status in children referred to a pediatric immunization clinic on account of egg allergy. Hum Vaccin. 2005;1(3):118-22. doi: 10.4161/hv.1.3.1888. [PubMed: 17012862].

25. Patja A, Davidkin I, Kurki T, Kallio MJ, Valle M, Peltola H. Serious adverse events after measles-mumps-rubella vaccination during a fourteen-year prospective follow-up. Pediatr Infect Dis J. 2000;19(12):1127-34. [PubMed: 11144371].

26. Tuncel T, Sancakli O, Ozdogru E. Successful administration of measles-rubella-mumps vaccine by graded challenge in a case with anaphylaxis after prior vaccination. Arch Argent Pediatr. 2017;115(2):e89-91. doi: 10.5546/aap.2017.eng.e89. [PubMed: 28318190].

27. Patja A, Makinen-Kiljunen S, Davidkin I, Paunio M, Peltola H. Allergic reactions to measles-mumps-rubella vaccination. Pediatrics. 2001;107(2). E27. doi: 10.1542/peds.107.2.e27. [PubMed: 11158501].

28. Fox A, Lack G. Egg allergy and MMR vaccination. Br J Gen Pract. 2003;53(495):801-2. [PubMed: 14601358]. [PubMed Central: PMC1314715].

29. Hawkes CP, Mulcair S, Hourihane JOB. Is hospital based MMR vaccination for children with egg allergy here to stay? Ir Med J. 2010;103(17-9). 